\title{
The Driver Fatigue Status Classification Based On Multi Region Evidence Support
}

\author{
Ye Sun \\ College of computer, Changchun Normal University, \\ Jilin 130032, China \\ Sunye_0406@sina.com
}

\begin{abstract}
Multi-observation areas based ensemble learning models are proposed for general fatigue performance identification. It is believed that although the expression of fatigue is not obvious. If the observation areas are concerned, the performance of fatigue will be relatively concentrated and the law of expression changes will be much clearer. Another advantage of feature extraction from the observation areas is that it can greatly reduce the interference caused by redundant information in the face when learning classifiers. For each observation area, a C4.5 base classification model is built. Each base model is equivalent to an independent decision maker. But due to its limited capacity, it may not be able to give accurate decisions. However, if the information provided by each decision maker is combined together, it will form comprehensive evidence. Driver status classification results given by ensemble learning approach are more accurate and stable.
\end{abstract}

Keywords: computer vision, fatigue driving, rough set, feature selection, ensemble learning

\section{Introduction}

Fatigue is a very complex physical appearance, and if an expression of fatigue as a human point of view, this expression is very subtle and difficult to grasp. This is the reason that the fatigue performance in general difficult to identify. However, for the driver fatigue monitoring, can identify more fatigue performance in general, to earlier fatigue warning significance will be very great. In the actual environment, because the vehicle is often in high-speed motion state, short fatigue may have caused dangerous, not to mention the typical fatigue performance.

In fact, some perceived slight signs such as frowning face, mouth slightly open, eye movement retardation, downcast mouth are related to the occurrence and fatigue. Most of the time in some combination of details is sufficient to note that the people have a sense of fatigue. If the combination of the multiple observation points is described and judgment on the driver's state, is bound to be more reasonable and reliable.

\section{Multi-level Information Acquisition}

All the features including local observation of regional features and global observation of regional features, here introduces the observation area, low dimensional manifold features by locally linear embedding get the globe.

Global observation area contains the core part of the face region. It is also a high dimension data. This paper hopes to get a macro sense from the high dimensional data, hidden behind the essence of high dimensions by a handful of hidden variables determined by the driver status description. This work is actually looking for high dimensional data manifold low dimensional embedding problems. It is often said that the popular learning. 
Manifold learning is a method of nonlinear dimension reduction. Dimension reduction methods can be divided into linear and nonlinear methods. Analysis of the widely used method including principal component analysis (PCA), linear discriminant analysis (LDA) and multiple discriminant analysis (MDA) belongs to the category of linear dimension reduction.

In 1990 Kirby [1] will be the first principal component analysis is introduced to the field of pattern recognition, Turk [2] are extended to this thought, and to develop a feature description based on subspace transforms the way. For a given the optimization object function, by a linear or nonlinear space transformation to obtain high dimensional data in a low dimensional subspace of the compact expression, reduce the computational complexity and achieve better classification results. Among them, PCA is looking for the performance of spindle orientation distribution of samples, which mainly deal with the sample data of Gauss distribution. LDA is a kind of supervised dimensionality reduction method. It is looking for is to make the divergence between class $s_{b}$ and class $s_{w}$ to reach maximum subspace. MDA is a LDA extension to the multi class case, processing is more complex, for problem $c$ class, MDA is projecting the sample to dimensional linear space of $c-1$. Three kinds of linear dimension reduction method are shown in Figure 1 .
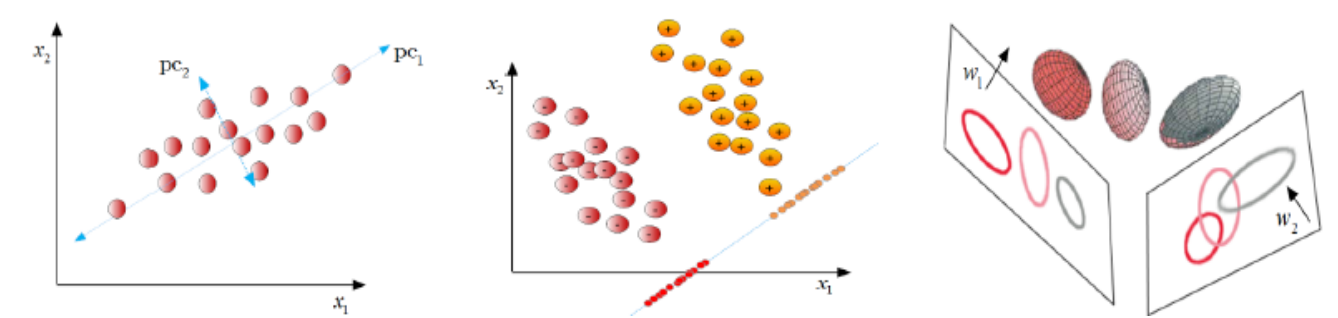
a) Principal Component Analysis b) Linear Discriminant Analysis c) Multiple Discriminant Analysis

Figure 1. Three Linear Dimensionality Reduction Methods

\section{Feature Evaluation Method Based On Rough Set}

This paper will introduce the kernel fuzzy rough set method to filter approach to feature selection and evaluation [3], from the candidate features and each feature subset obtained most related classification tasks.

Granulation and approximation are the cornerstone to construct the rough set theory, granulation performance in cognitive concept formation by inductive reasoning process approximation; embody the concept of interpretation and cognition to the concept of the unknown. Because of the difference of grain evolution of a rough set model is different, the classical Pawlak rough set is based on equivalence relations above, in addition to neighborhood relations are established based on rough set, the ordering relation established dominance based rough set and the following will be presented based on fuzzy equivalence relation is established based on the fuzzy rough set.

Fuzzy rough set model human cognition and reasoning, uncertainty in data analysis is widely used by [4-5]. Fuzzy rough set theory is as a mathematical tool to deal with uncertainty information package with two types of uncertainty, namely, rough granulation of ambiguity and approximation [6-7]. In dealing with uncertain information shown strong ability [8-9], and formatted in a mature computing framework [10].

The basis of the model is the decision consistency assumption: if the fuzzy sample decision category in the same neighborhood classification is consistent, otherwise, the classification of the sample is not consistent with the sample to the nearest heterogeneous sample distance to measure the distance, the higher the greater the degree of consistency of the sample classification. Based on fuzzy rough set assumptions can be seen, it focuses 
on the sample interval in the calculation process, the fuzzy rough set model feature selection embodies the structural risk minimization principle, therefore, this model for feature processing complex high dimensional data selection problem more effectively.

This paper constructed a feature evaluation and selection algorithm based on fuzzy rough set model, and experiment showed that selection feature subset is rationality and validity.

Given a fuzzy approximation space $\langle U, A, D>, B \subseteq A$. $\mathrm{R}$ is a fuzzy relation of the sample by the attribute subspace induced. Then, the decision $D$ on attribute subspace dependency based on two different approximate operators $S$ and $\theta$ are defined as:

$$
\begin{array}{r}
\gamma_{B}^{S}(D)=\frac{\left|\bigcup_{i=1}^{m} R_{S} d_{i}\right|}{|U|} \\
\gamma_{B}^{\theta}(D)=\frac{\left|\bigcup_{i=1}^{m} R_{\theta} d_{i}\right|}{|U|}
\end{array}
$$

This paper designs a feature selected prior to the greedy search algorithm, the algorithm is described as follows:

Algorithm1 Feature Selection based on Fuzzy Rough Sets

INPUT: $\langle U, A, D\rangle, K . U$ is sample set, $A$ are the condition attributes. $D$ is decision attribute, $\mathrm{K}$ implied in $\gamma$ computing, is a equivalence relations of satisfy $T$ the similarity measure functions

OUTPUT: Feature subset $F$

$1 F \leftarrow \varnothing$

2 While $A-F \neq \theta$ do

3 Foreach $\alpha_{i} \in A-F$ do

4 Computed $\gamma_{F \cup a_{i}}(D)$

5 End

6 Select $\alpha \in A-F$ subject to $\gamma_{F \cup a_{i}}(D)=\max _{i} \gamma_{F \cup a_{i}}(D)$

7 If $\gamma_{F \cup a_{i}}(D)-\gamma_{F}(D) \leq \varepsilon$ then

8 Suspend while cycle

9 End

$10 F \leftarrow F \cup a$

11 End

12 Return F

\section{Pattern Classifier Integration}

\subsection{C4.5 Decision Trees}

ID3 (Iterative Dichotomizer3) [11] and C4.5 [12] are the two most important decision tree learning algorithm, two algorithms are designed by Quinlan. The $\mathrm{C} 4.5$ algorithm can also be considered for the ID3 algorithm based software extensions, aims to address some of the issues of ID3 algorithm cannot handle. C4.5 algorithm inherits the advantages of ID3 algorithm, while the C4.5 decision tree like ID3, is constructed decision tree based on the information entropy of the concept in the training data set, but the $\mathrm{C} 4.5$ algorithm in the following 5 aspects of the ID3 algorithm is optimized:

(1) C4.5 is based on information gain rate instead of ID3 to achieve attribute selection, avoid the multi value attributes information gain results tend to choose property problem. 
(2) C4.5 is by creating the threshold can be the discretization of continuous attributes, and ID3 cannot deal with continuous attributes.

(3) C4.5 used effective pruning strategies, enhance the generalization performance of the decision tree, avoid over fitting phenomenon.

(4) C4.5 is able to attribute missing samples were extended, which can handle property incomplete data.

(5) C4.5 can be generated decision tree based on knowledge representation and eventually formed the production rules.

\subsection{The Base Classifier Ensemble Learning}

Ensemble learning is also known as combination, aggregation, committee and classifier fusion, refers to the comprehensive use of a group of classifier is used to classify a problem, in order to achieve better performance than the single classifier. If a classifier to a decision maker, process ensemble learning is equivalent to multiple decision-makers in decision-making.

Effectiveness of ensemble learning can be calculated and expressed three aspects are explained from the statistics, correlation analysis, Dietterich [13] and Kuncheva [14] are correlation analysis to explain. Assuming the space is $H, D_{i}$ is the set of samples obtained by the model of learning, $D^{*}$ is the best hypothesis of the problem.

(1) Statistical explanation, it is shown in Figure 2.

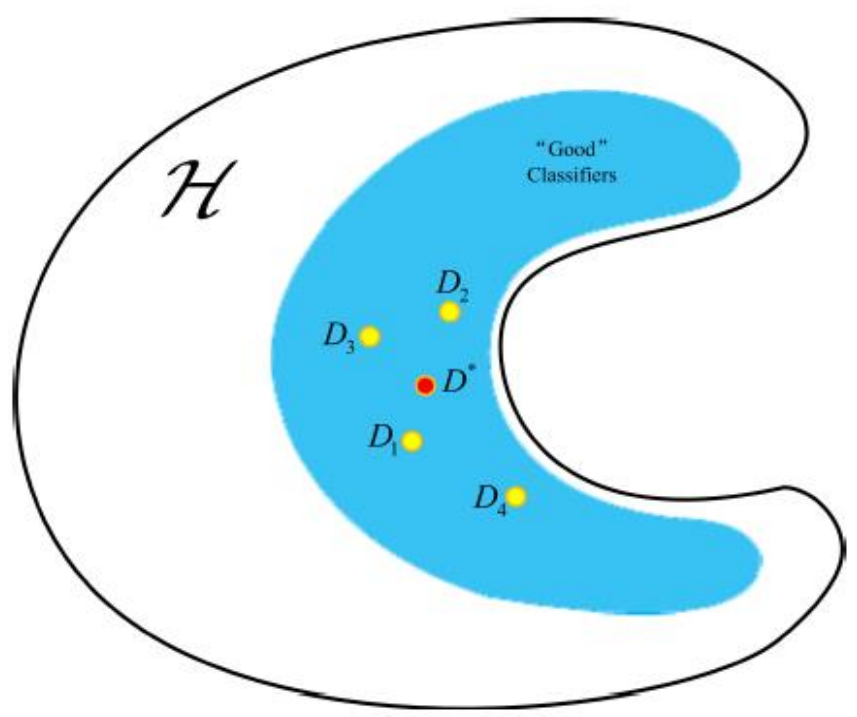

Figure 2. Statistical Reason for the Role of Ensemble Learning

The learning process is the process for the optimal hypothesis in the hypothesis space, when the training set is relatively hypothesis space is small, can learn a set of performance is generally better classifier from the training set, if from a randomly selected as classifier is used, then there is a definite risk will be one of the more poor classifier. But if the average output of this set of classifiers as classification result would be a safer choice, firstly because of the generalization ability of the classifier is different and may complement each other, the classification results is probably a better performance than that of single classifier. Even if the results did not give optimal performance of this classifier in the result is good, but it avoids the risk to poor classifier, thus ensuring the classification performance is stable and reliable. 
(2) Computational explanation, it is shown in Figure 3.

A learning model for optimal solution is a NP-hard problem, often need to introduce heuristic strategies, such as hill climbing method, greedy strategy and random search strategy, to reduce the complexity of the model, but the results of this study have a local optimal solution. The learning process starts by assuming a position hypothesis space. Learning should be located near the end of the optimal hypothesis. If these different local optimal solution to integration, so is possible to that some integration results than any single classifier are more optimal hypothesis.

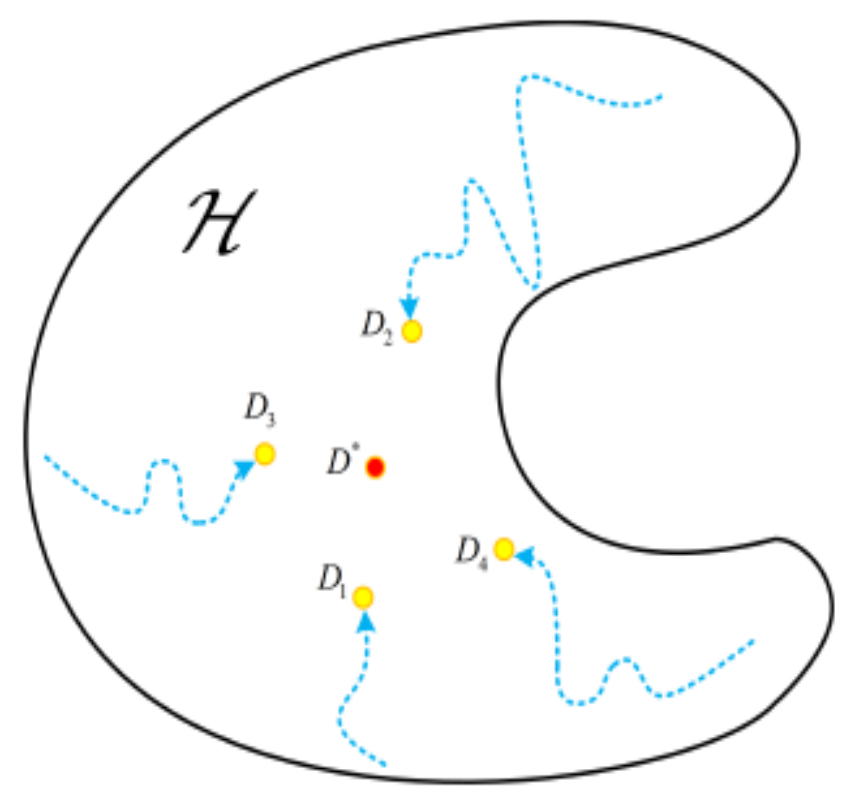

Figure 3. Computational Reason for the Role of Ensemble Learning

(2) Representational explanations, it is shown in Figure 4.

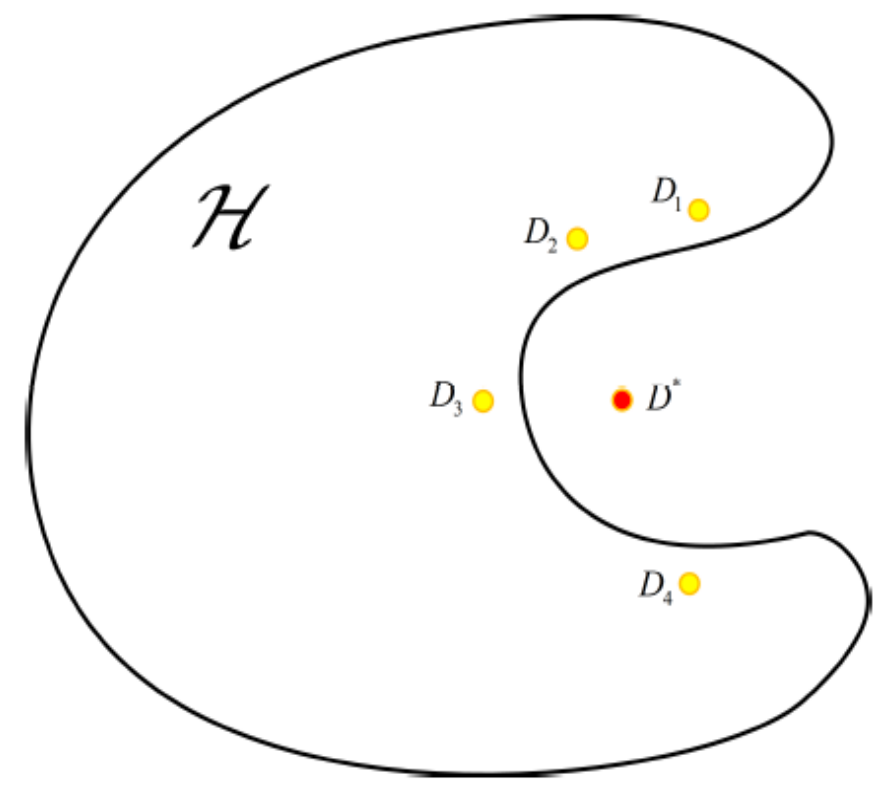

Figure 4. Representational Reason for the Role of Ensemble Learning 
In the learning process, real objective function is not in the hypothesis space pre-setting, such as a data set of the optimal hypothesis is a nonlinear classifier, if the hypothesis space is limited in linear classifier, then no matter how are unlikely to get the optimal classifier, which is that the optimal classifier is not in default in the hypothesis space. However, linear classifier, the combination can any preset accuracy approximation of any decision boundary.

\section{Experiment Design and Discussion}

Experimental data took driving video of 18 different positive simulated images in the experimental environment. Video frames is 11814 frames. Each video contains some fatigue performance. After correction and normalization of the face region expression sequence is shown in Figure 5 segmentation in video. Figure 6 shows a feature of a number of different fatigue unit.

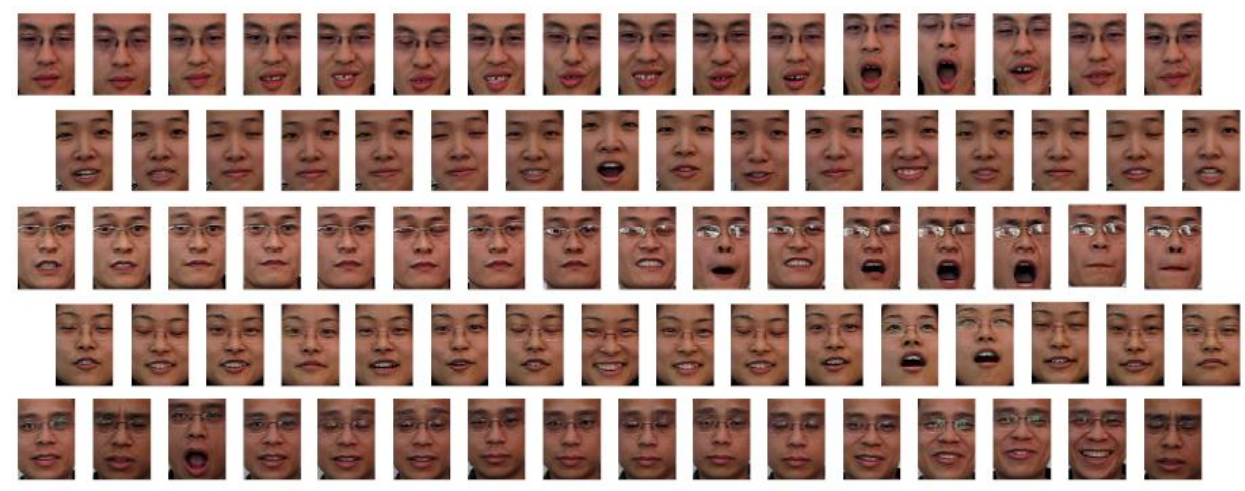

Figure 5. Face Expression Sequences of Different Drivers

The overall unit can obtain 45 sequences. Obtains 36 sequences from each local unit. Figure 7 shows some closely related to fatigue performance of the sequence features.

The global fatigue unit as an example, Figure 8 shows the characteristics of each single decision dependence. All 9 fatigue unit single feature decision dependent, it is shown in Figure 9.

As previously mentioned, all features are not necessary. In each fatigue unit only select a few important features to form feature subset which based classification model. According to each unit feature subset decision dependent variation degree, this paper finally from each fatigue unit selects 15 characteristics of the driver's state. Table 1 and Table 2 describe the characteristics of various fatigue unit selection.

In table1, most feature is the arrangement sequence features and characteristics of a certain consistency. Select results from the characteristics of the global unit of Unit1, characteristics of the 45 is a feature of most discriminating, it shows the waveform factor LLE an embedding dimension of (Shape Factor), this means change and fatigue expression the dimension has a significant correlation. 


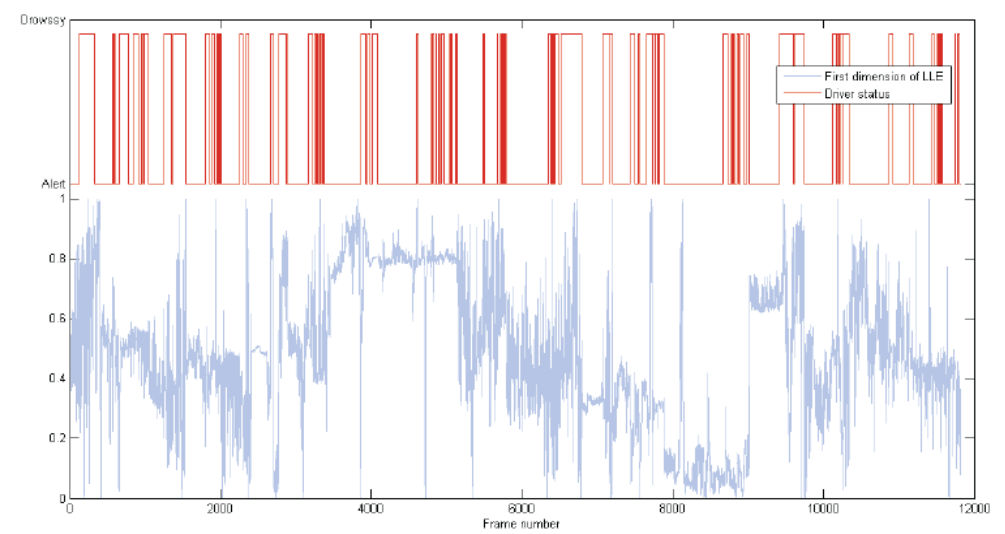

a) LLE's First Dimension of Global Fatigue Unit

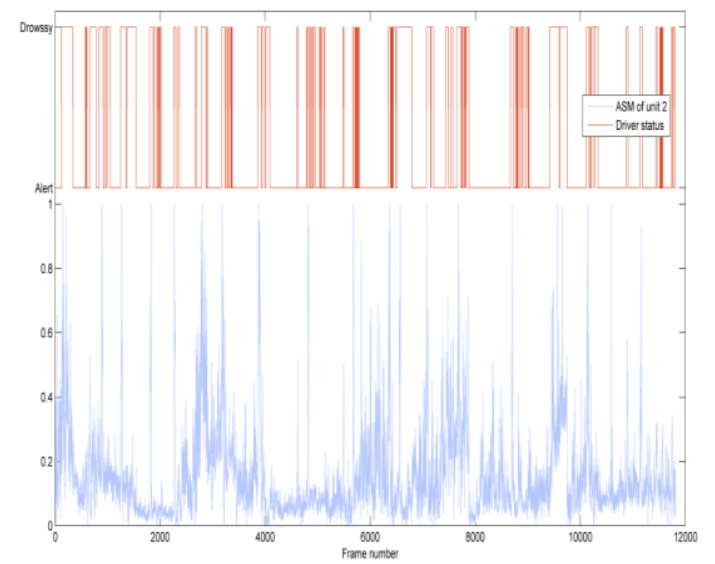

b) Angular Second Moment of Fatigue Unit 2

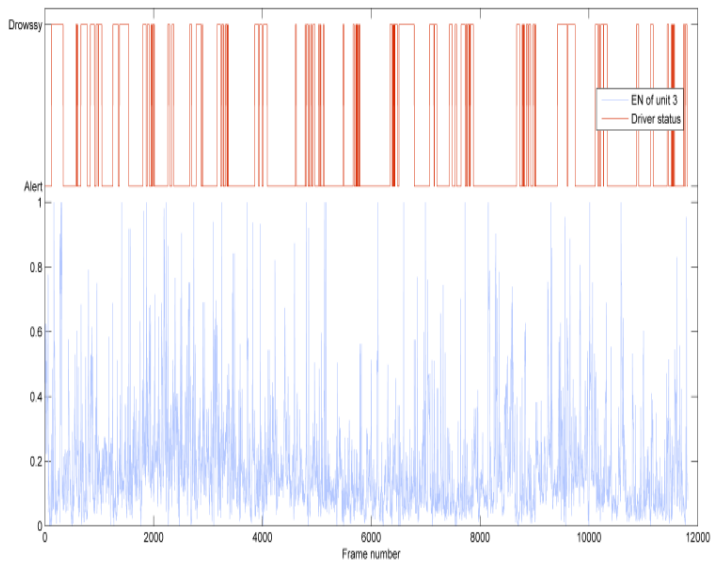

c) Entropy of Fatigue Unit 3 


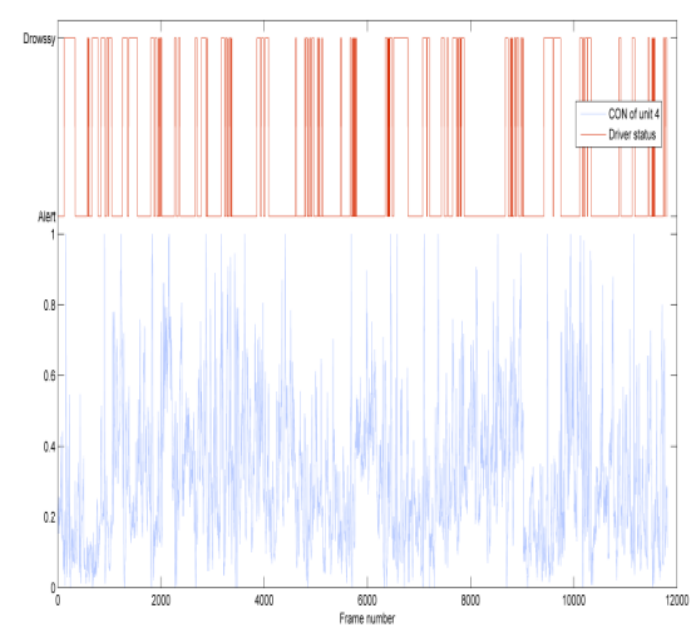

d) Contrast of Fatigue Unit 4

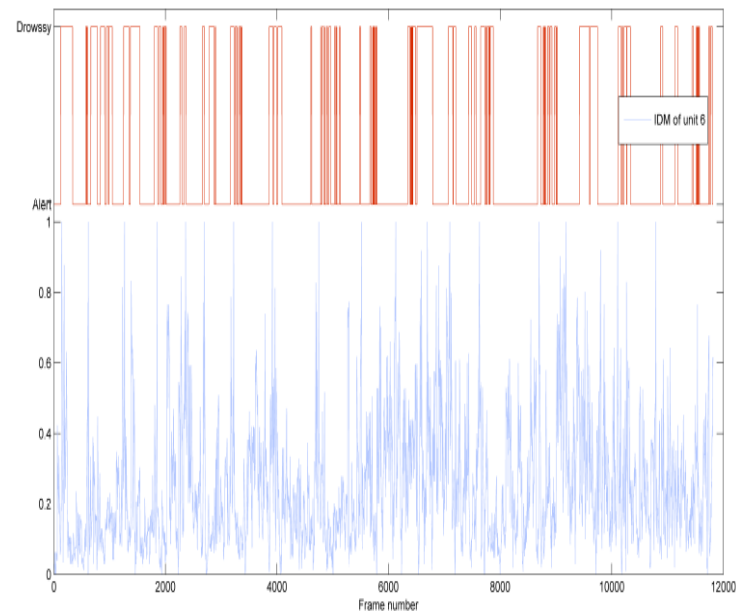

e) Inverse Difference Moment of Fatigue Unit6

\section{Figure 6. Static Information from Different Fatigue Units}

In each fatigue unit change with characteristics of sequentially adding decision dependence is shown in Figure 10. Figure 11 shows the complementary global unit selected feature is most prominent. Fuzzy decision final feature subset of 15 features of dependence is up to 0.9338 .

From Figure 10 also can draw a conclusion, all fatigue unit selects a feature subset is good, first of all, all units feature subset generated decision dependency has a higher value, this paper set up regional observation is closely related with the fatigue expression, and characterization of feature subset of its own have very strong upward trend; secondly, each curve in the graph shows obvious consistency, that feature subset each unit has a consistent description ability for fatigue expression occurs, it is beneficial for the performance model set to improve. Further, the distribution histograms to calculate the local fatigue unit feature, it is shown in Figure 11.

In Figure 11, sequence features more clearly are relatively important, with the exception of a few static features, feature selection results mainly concentrated in the sequence features. Characteristics of the 21 is the contrast, which is the highest frequency of static characteristics is selected. Contrast is a reflection of a measure of image clarity, at the same time, it can also describe the texture depth. If the texture is deep and clear, will be to a large value contract. Conversely, if the image is fuzzy or no obvious texture, contract value will be small. In the gray co-occurrence matrix, the greater difference between pixels, contrast value will be greater. Important facial expression such as texture 
information recording the driver is useful, the folds of the skin caused by fatigue expression such as can be described by contrast.

The most important features of the sequence have mainly 4, label 14 entropy is respectively sequence characteristic, label 15 is characteristic of entropy sequence waveform factor, label 23 is contrast characteristic of standard sequence. Label 28 is contrast characteristics of sequence crest factor.

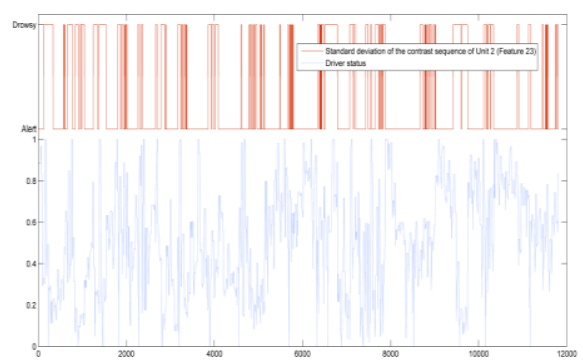

a) The $23^{\text {th }}$ feature of Fatigue Unit 2

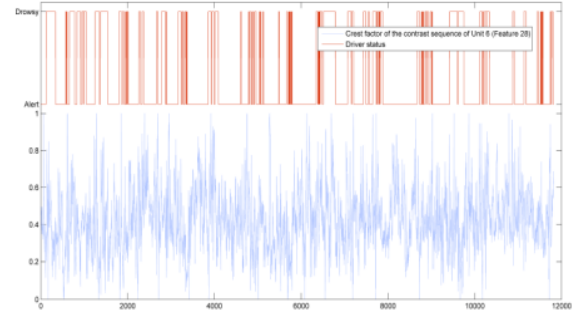

c) The $28^{\text {th }}$ feature of Fatigue Unit 6

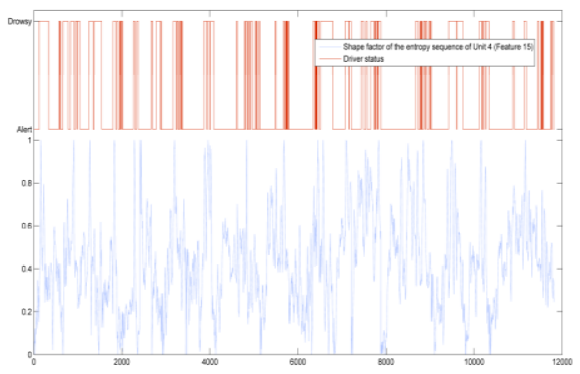

b) The $15^{\text {th }}$ feature of Fatigue Unit 4

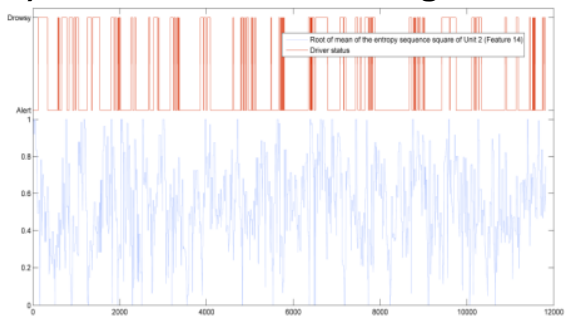

d) The $14^{\text {th }}$ feature of Fatigue Unit 8

Figure 7. Some Important Sequential Information from Different Fatigue Units

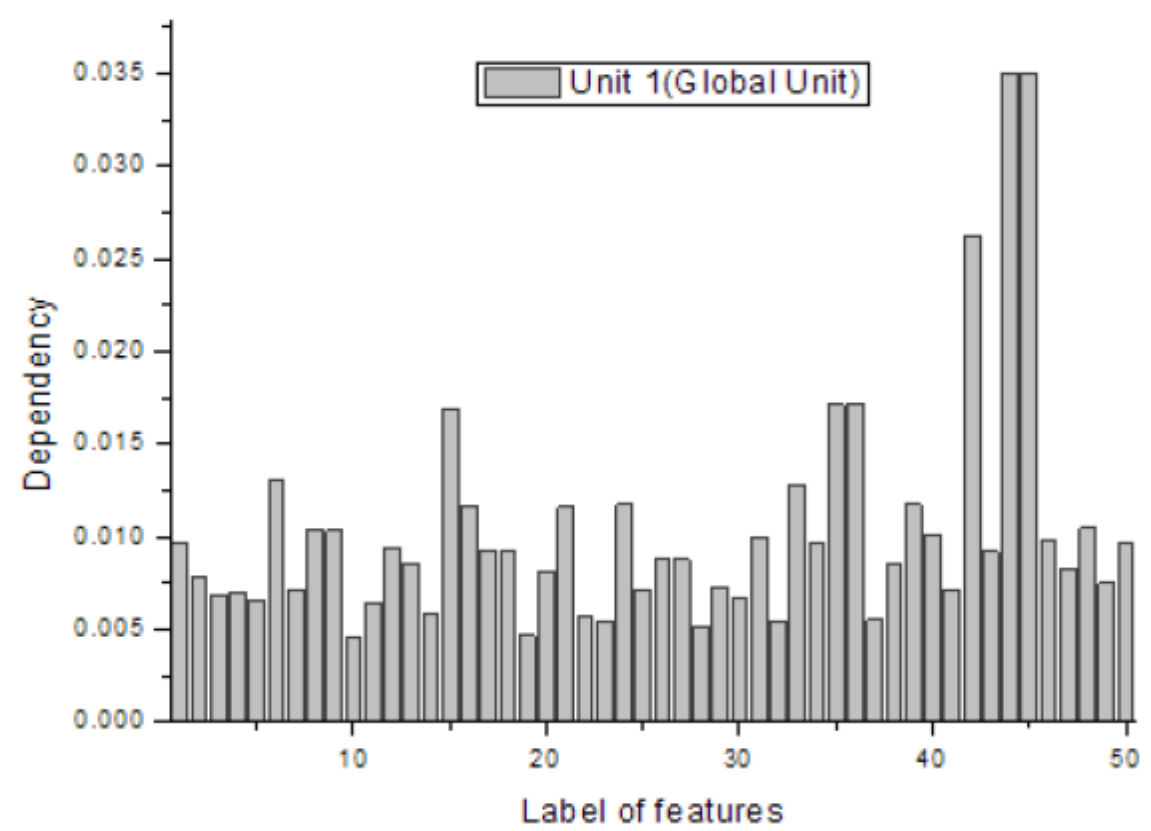

Figure 8. Dependency of Decision on Each Feature of the Global Fatigue Unit 


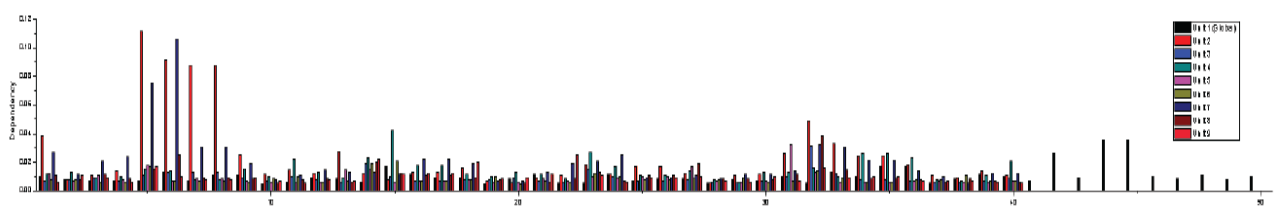

Figure 9. Dependency of Decision on Each Feature of All Fatigue Units

Table 1. The Features Selected from Each Unit

\begin{tabular}{|c|c|c|c|c|c|c|c|c|c|c|c|c|c|c|c|}
\hline \multicolumn{16}{|c|}{ Labeloffeatures } \\
\hline $\begin{array}{l}\text { Unit } \\
1\end{array}$ & 45 & 42 & 33 & 34 & 25 & 16 & 5 & 6 & 7 & 29 & 24 & 47 & 2 & 22 & 17 \\
\hline $\begin{array}{l}\text { Unit } \\
2\end{array}$ & 5 & 14 & 23 & 18 & 37 & 30 & 12 & 33 & 28 & 19 & 39 & 15 & 10 & 24 & 21 \\
\hline $\begin{array}{l}\text { Unit } \\
3\end{array}$ & 32 & 14 & 23 & 37 & 18 & 6 & 10 & 28 & 15 & 9 & 30 & 39 & 2 & 3 & 16 \\
\hline $\begin{array}{l}\text { Unit } \\
4\end{array}$ & 15 & 34 & 24 & 37 & 5 & 28 & 30 & 14 & 21 & 3 & 32 & 19 & 12 & 29 & 23 \\
\hline $\begin{array}{l}\text { Unit } \\
5\end{array}$ & 31 & 14 & 24 & 5 & 18 & 19 & 30 & 32 & 28 & 10 & 12 & 15 & 23 & 1 & 20 \\
\hline $\begin{array}{l}\text { Unit } \\
6\end{array}$ & 15 & 5 & 23 & 14 & 10 & 16 & 30 & 12 & 37 & 33 & 19 & 32 & 28 & 22 & 9 \\
\hline $\begin{array}{l}\text { Unit } \\
7\end{array}$ & 6 & 14 & 23 & 28 & 30 & 37 & 19 & 39 & 21 & 20 & 24 & 15 & 12 & 4 & 10 \\
\hline $\begin{array}{l}\text { Unit } \\
8\end{array}$ & 32 & 23 & 14 & 5 & 30 & 19 & 21 & 28 & 3 & 36 & 10 & 15 & 9 & 33 & 37 \\
\hline $\begin{array}{l}\text { Unit } \\
9\end{array}$ & 22 & 14 & 23 & 5 & 9 & 28 & 27 & 32 & 21 & 10 & 39 & 19 & 4 & 15 & 24 \\
\hline
\end{tabular}

Table 2. The Features Selected from Each Unit

\begin{tabular}{|l|l|l|l|l|}
\hline & $\begin{array}{l}\text { Original } \\
\text { Dimension }\end{array}$ & $\begin{array}{l}\text { Current } \\
\text { dimension }\end{array}$ & $\begin{array}{l}\text { Significance of } \\
\text { the most } \\
\text { important } \\
\text { feature }\end{array}$ & $\begin{array}{l}\text { Significance } \\
\text { of the selected } \\
\text { feature subset }\end{array}$ \\
\hline Unit1 & 4941 & 15 & 0.035059 & 0.933812 \\
\hline Unit2 & 480 & 15 & 0.111547 & 0.879897 \\
\hline U it3 & 416 & 15 & 0.031486 & 0.869181 \\
\hline Unit4 & 651 & 15 & 0.04187 & 0.824764 \\
\hline Unit5 & 288 & 15 & 0.031914 & 0.836349 \\
\hline Unit6 & 192 & 15 & 0.020525 & 0.836671 \\
\hline Unit7 & 480 & 15 & 0.105784 & 0.886796 \\
\hline Unit8 & 288 & 15 & 0.038313 & 0.874637 \\
\hline Unit9 & 192 & 15 & 0.025061 & 0.83755 \\
\hline
\end{tabular}

The above results can be seen, the strong ability of entropy has texture characterization. Entropy is a measure of the amount of image information, if the gray level co-occurrence matrix with random, that is to say, the symbiotic each element in the matrix is almost equal, then the entropy will be great. At the same time, entropy can reflect the nonconformance and complexity of image texture. Root mean square (RMS) reflects the sequence energy. Energy entropy sequence can well reflect the change of local texture. Therefore, characteristic 14 is a very good feature to distinguish the driver fatigue and waking states. 
In the above 4 features, characteristics of 23 and characteristics of 14 can provide changing information of local fatigue unit and, because the characteristics of 14 and 23 were selected out earlier is than 15 and 28 , so they are more important.

Next, need to verify the ensemble learning is effective, the base model of feature subset is integrated. Experiment with a classifier with the highest accuracy based model at the start, according to the classification accuracy from high to low to medium model, until all 9 base models are integrated.

The integrated model of different scale is also using by $90 \%$ off the cross validation method. The mean and the error bounds are given TPrate,TNrate and Precision of three evaluation indexes, the results are shown in Figure 12.

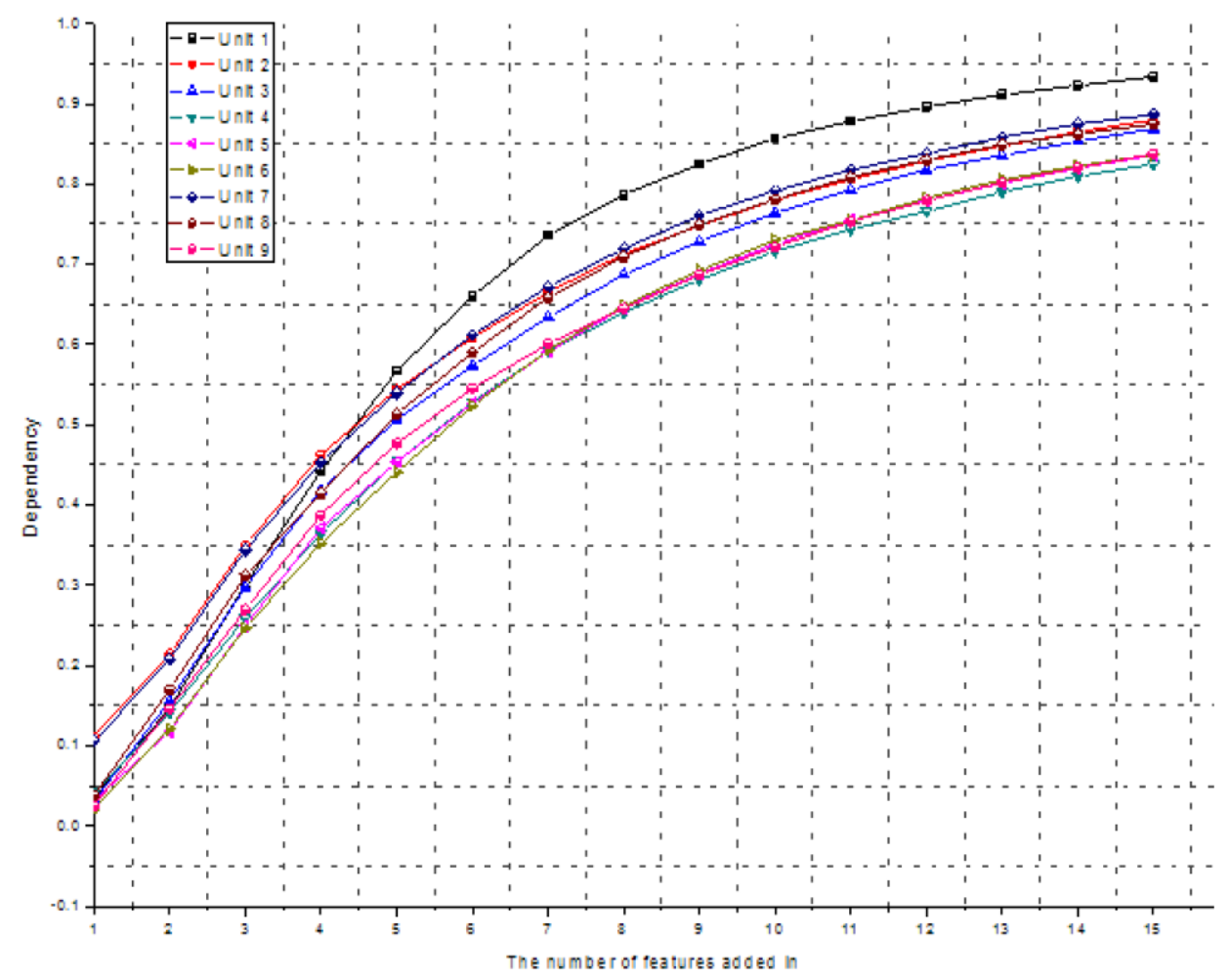

Figure 10. Dependency of Decision on Increasing Feature Subsets 


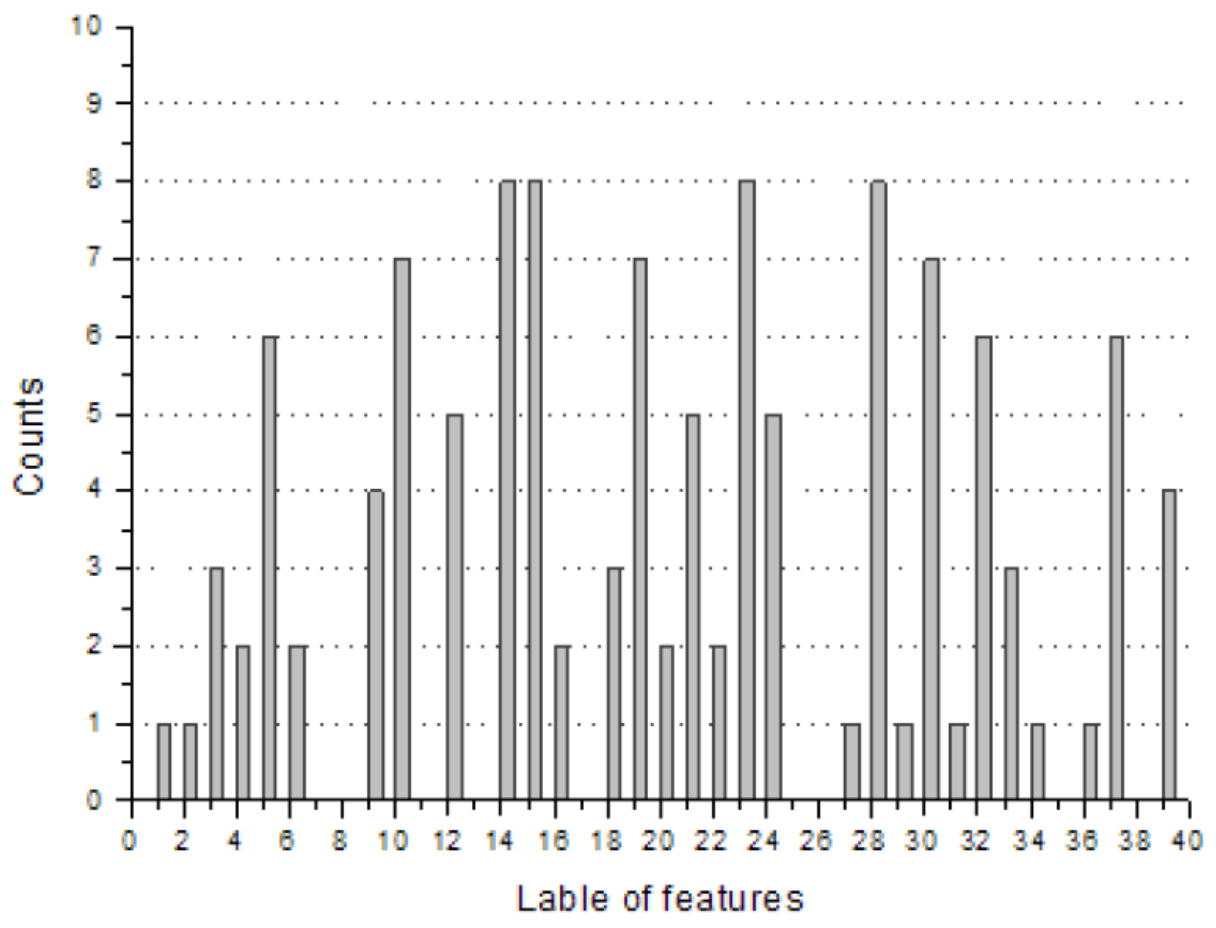

Figure 11. Histogram of the Features Selected from All Local Units

Through all the above findings. After feature selection based model can even get better classification results. If the 9 base model integrated the best classification accuracy can reach $97.55 \%$. Therefore, the experimental results also are very effective based on the feature selection of fuzzy rough sets and ensemble learning

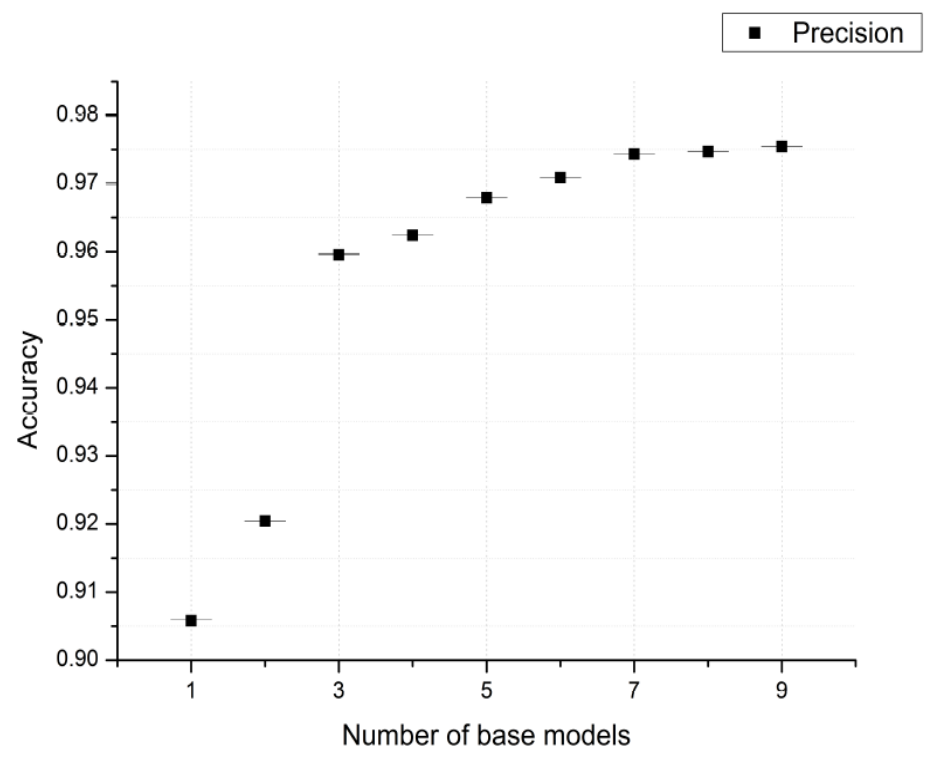

a) Changes in Classification Precision 


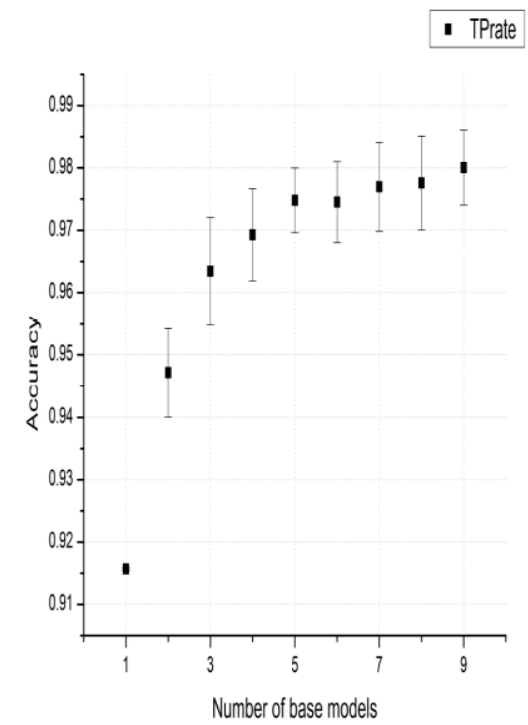

b) Changes in True Positive Rate

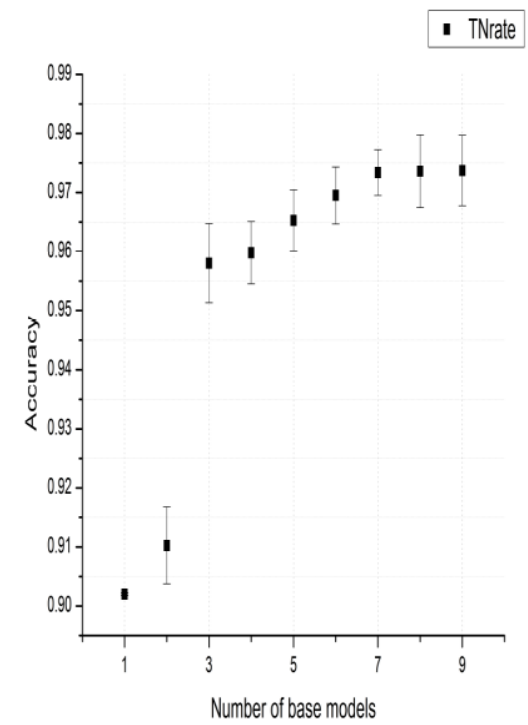

c) Changes in True Negative Rate

Figure 12. Comparison of Classification Performance with Different Number of Base Models Embedding

\section{Conclusion}

In this paper, the driver's facial information to identify the driver's state, there will be fatigued as complex facial expressions recognition, this is a challenging task, because the fatigue performance is much more subtle, difficult to describe. The formation of a human face image is a high dimension information space, recognition of fatigue expression, a key problem is how to extract the complementary features and eliminate the redundant information from the face region; Another key issue is to get information after the use of what kind of reasonable use of the various facial information.

The driver's state is usually through the whole face area shown, different people may have some differences, so the use of a single regional characteristics to determine the driver's state has certain difficulty. However, if the total face information using words and can easily lead to over fitting phenomenon occurs.

This work properly dealing with the contradictions between the two aspects, by setting the number of fatigue observation area, makes facial expressions related information to be more centralized. Considering that this information is static. So the introduction of sliding time window technique to obtain sequence information related to expression. Characteristic quality is reasonable evaluation by kernel fuzzy rough set technology, greatly reduce the dimension of feature space. The base model was based on C4.5 decision tree learning model to make good diversity and complementarity and achieved the expected results.

\section{References}

[1] M. Kirby and L. Sirovich, "Application of the Karhunen-lokve Procedure for the Characterization of Human Faces”, IEEE Transactions on Pattern Analysis and Machine Intelligence, vol. 12, no. 1, (1990), pp. 103-108.

[2] M. Turk and A. Pentland, "Eigenfaces for Recognition", Journal of Cognitive Neuroscience, vol. 3, no. 1, (1991), pp. 71-86.

[3] J. B. Tenenbaum, V. D. Silva and J. C. Langford, "A Global Geometric Framework for Nonlinear Dimensionality Reduction”, Science, vol. 290, no. 5500, (2000), pp. 2319-2323.

[4] X. Wang, E. C. Tsang, S. Zhao, et al., "Learning Fuzzy Rules from Fuzzy Samples Based on Rough Set Technique", Information Sciences, vol. 177, no. 20, (2007), pp. 4493-4514.

[5] Q. Hu, Z. Xie and D. Yu, "Hybrid Attribute Reduction Based on a Novel Fuzzy-rough Model and Information Granulation”, Pattern Recognition, vol. 40, no. 12, (2007), pp. 3509-3521. 
[6] L. Zhou and W. Z. Wu, "On Generalized Intuitionistic Fuzzy Rough Approximation Operators", Information Sciences, vol. 178, no. 11, (2008), pp. 2448-2465.

[7] S. Zhao, E. C. C. Tsang and D. Chen, "The Model of Fuzzy Variable Precision Rough Rets", IEEE Transactions on Fuzzy Systems, vol. 17, no. 2, (2009), pp. 451-467.

[8] D. Ciucci, "On the Axioms of Residuated Structures: Independence, Dependencies and Rough Approximations", Fundamenta Informaticae, vol. 69, no. 4, (2006), pp. 359-387.

[9] P. Maji and S. K. Pal, "Rfcm: A Hybrid Clustering Algorithm Using Rough and Fuzzy Sets", Fundamenta Informaticae, vol. 80, no. 4, (2007), pp. 475-496.

[10] B. Moser, "On Representing and Generating Kernels by Fuzzy Equivalence Relations", Journal of Machine Learning Research, vol. 7, (2006), pp. 2603-2620.

[11] J. R. Quinlan, "Induction of Decision Trees", Machine Leaming, vol. 1, no. 1, (1986), pp. 81-106.

[12] J. R. Quinlan, "C4.5: Programs for Machine Learning”, San Mateo, California, USA: Morgan Kaufmann Publishers, (1993).

[13] T. G. Dietterich, "Ensemble Methods in Machine Learning", Proceedings of the 1st International Workshop on Multiple Classifier Systems (MCS 2000). London, UK, (2000), pp. 1-15.

[14] L. I. Kuncheva, "Combining Pattern Classifiers: Methods and Algorithms", Hoboken, NJ: John Wiley \& Sons Inc., (2004).

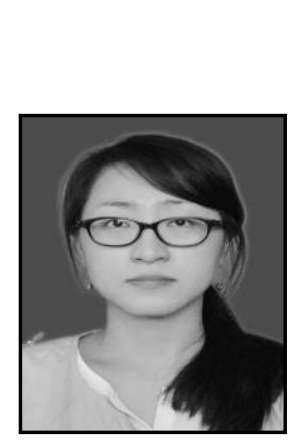

\section{Author}

Sun Ye, she is a lecturer college of computer in Changchun Normal University. She is in the research of computer application. 